\title{
Riesgo físico y enfermedades profesionales en trabajadores que operan equipos de vibración en construcciones civiles.
}

\section{Physical risk and professional diseases in workers who operate vibration equipment in civil constructions}

\author{
Autor \\ Luis Alfredo Ormeño Bazurto. Graduado de Maestría de Seguridad y Salud Ocupacional \\ "Segunda Cohorte" de la Universidad San Gregorio de Portoviejo. Manabí. Ecuador. \\ E-mail:inglaob67@hotmail.com
}

Fecha de recibido: 2019-04-29

Fecha de aceptado para publicación: 2019-12-23

Fecha de publicación: 2019-12-30

\section{Resumen}

Los Riesgos Físicos y Enfermedades Profesionales presenta un conjunto de definiciones y criterios médicos de tipo jurídicos y técnicos ajustados a la higiene industrial, donde la prevención de riesgos laborales juega un rol importante El objetivo de este estudio es describir el riesgo físico y enfermedades profesionales en trabajadores que operan equipos de vibración en construcciones civiles. Como metodología se adoptó la revisión sistemática exploratoria con enfoque cualitativo que orienta a realizar una revisión bibliográfica sintetizada, sobre los riesgos y posibles enfermedades ocupacionales provocadas por exposición a las vibraciones en el lugar de trabajo; el método bibliográfico, fue necesario para explicar teóricamente las variables estudiadas y esquematizar la información. El estudio se basa en la Norma ISO 5349 (medida de vibraciones mano-brazo) y la ISO 2631 (de cuerpo entero). Concluye la investigación a partir de la síntesis de los resultados obtenidos, dando respuesta a lo que respecta a materia de Riesgos Físicos y enfermedades profesionales en trabajadores que operan equipos de vibración, para lo cual se requiere que los Estados impulsen políticas preventivas que orienten las actuaciones de este grupo humano.

Palabras clave: Riesgos ergonómicos, trastorno musculo esquelético, vibraciones mano-brazo y cuerpo entero.

\begin{abstract}
The Physical Risks and Occupational Diseases presents a set of definitions and medical criteria of legal and technical type adjusted to industrial hygiene, where the prevention of occupational risks plays an important role The objective of this study is to describe the physical risk and occupational diseases in workers that operate vibration equipment in civil constructions. As a methodology, the systematic exploratory review was adopted with a qualitative approach that guides a synthesized bibliographic review of the risks and possible occupational diseases caused by exposure to vibrations in the workplace; The bibliographic method was necessary to theoretically explain the variables studied and schematize the information. The study is based on ISO 5349 (measurement of hand-arm vibrations) and ISO 2631 (full body). The investigation concludes from the synthesis of the results obtained, responding to the matter of Physical Risks and occupational diseases in workers operating vibration equipment, for which it is required that the States promote preventive policies that guide the actions of this human group.
\end{abstract}

Key words: Ergonomic risks, musculoskeletal disorder, hand-arm and whole-body vibrations. 


\section{Introducción}

Desde el origen de la humanidad las obras de construcción han sido de mucha importancia para la protección y subsistencia de los seres humanos. En la actualidad para el levantamiento de estas obras resulta determinante la interpretación de los procedimientos dados para tal objetivo. Desde la promulgación de la Ley de Prevención de Riesgos Laborales (1995) y sus reglamentos, aun se sigue intentando conseguir una legislación que permita mejorar las condiciones de los trabajadores y proteger su salud laboral.

Las Directivas europeas, en referencia a la seguridad y salud, establece su base jurídica en el artículo 153 del Tratado de Funcionamiento de la Unión Europea (UE), en su antiguo artículo 137 del TCE, en la que se autoriza a la propia UE a adoptar Directivas en este ámbito. Cualquiera de los Estados miembros será libre para imponer y proponer Normas para la protección y seguridad laboral de los trabajadores; sin embargo, se exige respetar el mínimo exigido por la Directiva, con la intención de evitar o minimizar las causas que generan los accidentes laborales y las enfermedades profesionales.

Este ordenamiento cuenta con un conjunto de normas, lo cual lo sintetizan de acuerdo a las siguientes estructuras: Normas Internacionales, (Convenios, OIT, Tratados internacionales); Normas Comunitarias (Directivas); Legislación Estatal (Leyes, Normas Técnicas); y, otras fuentes (Convenios colectivos, normas autonómicas o locales). Muchos países consideran la adopción de normas o directrices sobre exposición a vibración transmitidas a las manos y se basan a la Norma ISO 5349, misma que recomienda el uso de una curva de ponderación de frecuencia que proporcione un valor aproximado de la sensibilidad de la mano a los estímulos de vibración dependiente de una frecuencia.

El sector de la construcción posee las más altas tasas de accidentes que provocan enfermedad, discapacidad y muerte. De Vicente Abad (2006) expresa que: "El sector de la construcción es el segundo con mayor Índice de Incidencia de enfermedades profesionales, después del sector industrial" (p. 21).

Conocer las condiciones de los trabajadores de la construcción es importante, debido a que este se ha convertido en una necesidad vital para la subsistencia de los seres humanos. Ante esta situación el presente estudio tiene como objetivo determinar el riesgo físico y enfermedades profesionales a las que se exponen las personas que trabajan con maquinarias y equipos de construcciones civiles. Según el Instituto de Seguridad Laboral, Ministerio del Trabajo y Previsión Social (2014) las enfermedades profesionales son "causada de manera directa, por el ejercicio del trabajo que realice una persona y que le produzca incapacidad o 
muerte. $[\ldots]$, debe existir una relación causal entre el quehacer laboral y la patología que provoca la invalidez o la muerte" (p. 2).

Datos recopilados de documentos internacionales no reflejan resultados estadísticos enfocados directamente con acontecimientos o enfermedades implicadas con las vibraciones, pero si se presentan diagnósticos de dolencia en dedos, manos, muñecas, brazos codos, hombros y nuca. No existe un número de pacientes diagnosticados directamente por trastornos de vibraciones, ya que las actividades extra laborales pueden afectar más que la misma actividad profesional (Luttmann, Jarger, \& Griefahn, 2004). Se asume que, por realizar dichas actividades de manera constante por un determinado tiempo, se corre el riesgo de adquirir enfermedades profesionales.

\section{Metodología}

La metodología de este trabajo científico es de revisión sistemática exploratoria, que, según Manchado, Tamames, López, Mohedano; D'Agostino y Veiga (2009) "son trabajos que resumen de forma sistemática la evidencia científica para estudiar una relación existente en el ámbito de la salud contestando a una pregunta concreta" (p. 14). Previo a la revisión sistemática como tal, se estableció un proceso de análisis general de la información científica existente en las diferentes bases de datos sobre el tema que se desea conocer.

Se realizó un análisis de los diferentes temas relacionados con las palabras clave Riesgos ergonómicos, trastorno musculo esquelético, vibraciones mano-brazo y cuerpo entero. Con la finalidad escoger diversas publicaciones en el ámbito de la construcción por ser el tema de interés para el estudio sistemático.

La búsqueda fue direccionada a encontrar información en base de datos bibliográficas y buscadores de fuentes confiables como Web of Science, Scopus, CSIC y Dialnet, Latindex, Redalyc, Elsevier y SciELO, con fechas de publicación dadas en el periodo de 2001-2017, las mismas que direccionaron a los riesgos físicos y enfermedades profesionales en trabajadores que operan en equipos de vibración, con un total de 15700 publicaciones en español. Posteriormente en la segunda recopilación se fueron agregando las palabras de trastorno musculo esquelético, se redujo la brecha de investigaciones a 3245 publicaciones. Tomando en cuenta que el número de publicaciones era relativamente extenso se procedió añadir las palabras clave faltantes Riesgos ergonómicos, trastorno musculo esquelético, vibraciones mano-brazo y cuerpo entero dentro de la búsqueda para preseleccionar alrededor de 58 artículos de las 1529 publicaciones posibles con relación a las palabras clave. 
Se seleccionaron en la tercera búsqueda las más actuales a la fecha 2019 o de los últimos 10 años, inicialmente 58 artículos y almacenándolos en bases de datos, a estos se les aplicó la técnica de la cala de libros y con lo cual se pudo constatar el cumplimiento de los criterios de inclusión como artículos de estudios primarios (Merino Trujillo, 2011); artículos escritos en idioma inglés y español; artículos que contenían similitudes con las palabras clave relacionadas con riesgo físico y enfermedades profesionales. Los otros artículos tratan la temática de manera general en cuanto a los equipos de vibración en construcciones civiles.

Después se procedió a escoger ciertos artículos con criterios de exclusión: enfermedades profesionales que no son causadas por los equipos de vibraciones; Tesis de Pregrado; población que no usa equipos de vibración. Así mismo se obviarán datos no confiables donde la investigación haya sido radicada en un solo puesto de trabajo sin tomar en cuenta las actividades extra laborales presentadas en documentos investigados.

A partir de los 58 artículos se filtró aún más la búsqueda lo que permitió identificar los artículos que se relacionaban de forma más directa a la temática quedando un total de 30 y se excluyeron 28. A partir de esto se realizó el análisis bibliométrico tal como lo describe Manchado et al., (2009), en la cual se debe considerar de forma estricta datos de autores, año, país, idioma, así como también del contenido como: metodología, población y muestra, objetivos, resultados, conclusiones y referencia.

Esta investigación se la realizó en base a documentos con datos cualitativos y cuantitativos sobre afectaciones en la salud causados por vibraciones. Se buscó la credibilidad del escritor y se dio preferencia a los artículos que han tenido mayor cantidad de citas bibliográficas al igual que libros o aportes científicos internacionales. Datos cuantitativos por parte de entidades públicas o privadas que hayan sido expuestas o de libre acceso.

\section{Resultados}

Se seleccionaron publicaciones de varios países, de estos se pudo recabar información relevante a nivel mundial y de forma cercana en países de la Región Andina. A continuación, se presentan los resultados obtenidos.

Tabla 1. Publicaciones

\begin{tabular}{lcc}
\hline País & $\mathbf{N}^{\circ}$ de artículos & $\mathbf{\%}$ \\
\hline España & 7 & 23 \\
\hline México & 3 & 10 \\
\hline Ecuador & 2 & 7 \\
\hline Perú & 4 & 13 \\
\hline Venezuela & 3 & 10 \\
\hline Uruguay & 2 & 7 \\
\hline
\end{tabular}




\begin{tabular}{lcc}
\hline Argentina & 2 & 7 \\
\hline Colombia & 2 & 7 \\
\hline Cuba & 1 & 3 \\
\hline Alemania & 1 & 3 \\
\hline Australia & 1 & 3 \\
\hline Chile & 1 & 3 \\
\hline Canadá & 1 & 3 \\
\hline
\end{tabular}

Fuente: Datos obtenidos en la revisión sistemática exploratoria.

Los artículos estudiados fueron agruparon y clasificados de acuerdo a las variables, considerando sus atributos recurrentes (Kitchenham, 2004) donde se establece lo siguiente:

Tabla 2. Clasificación de artículos analizados por medio de las variables de estudios.

\begin{tabular}{|c|c|c|}
\hline Variables & Artículos que contienen las variables & $\begin{array}{c}\mathrm{N}^{\circ} \text { de } \\
\text { artículos }\end{array}$ \\
\hline $\begin{array}{l}\text { Riesgo físico y } \\
\text { enfermedades } \\
\text { profesionales }\end{array}$ & $\begin{array}{l}\text { (Solís Carcaño, 2006); (Alcantara, 2014); (Andrade y Gómez, } \\
\text { 2008); (Arenas y Cantú, 2013); (Bellorín, Sirit, Rincón y } \\
\text { Amortegui, 2007); (Calvo, 2014); (Collado, 2008); (De Vicente } \\
\text { Abad, 2006); (Echeverri y Yepes, 2011); (Fontaneda, Camino, } \\
\text { Manzanedo del Campo y Sáiz, 2005); (Gil Monte, 2012); (Guillén, } \\
\text { 2014); (Silva, 2001); (Velásco Mayor, 2013); (Viglioglia, 2008); } \\
\text { (Sanz, 2013); (Lobato, 2016). }\end{array}$ & $\begin{array}{c}\text { 17estudios (57 } \\
\%)\end{array}$ \\
\hline $\begin{array}{l}\text { Equipos de } \\
\text { vibración en las } \\
\text { construcciones } \\
\text { civiles que } \\
\text { generan daño a } \\
\text { los Trabajadores }\end{array}$ & $\begin{array}{l}\text { (Arias \& Martínez, 2017); (Arias, 2011); (Arias; 2012); (Arias y } \\
\text { Reyes, 2016); (Cardoso, Martínez, López y Arezes, 2013); (Instituto } \\
\text { Nacional de Seguridad e Higiene en el Trabajo, 2002); (Mugica, } \\
\text { Roman y Cadiz,.2004); (Peña; 2018); (Pichardo y Jiménez, 2007); } \\
\text { (Ramos y Abuja, (2014); (Santurio, Ferrera y López, 2003); (Weir y } \\
\text { Lander, 2005); (Wriu, 2012). }\end{array}$ & $\begin{array}{c}13 \text { artículos } \\
(43 \%)\end{array}$ \\
\hline
\end{tabular}

\section{Fuente: Datos obtenidos en la revisión sistemática exploratoria.}

En lo que respecta a la variable riesgo físico y enfermedades profesionales, se consultaron 17 artículos lo que representa el 57 \%; la variable equipos de vibración en las construcciones civiles que generan daño a los Trabajadores, fue analizada con 13 artículos correspondientes al $43 \%$ del total, estos hacen referencia a la protección, promoción y mejora de la salud de los trabajadores, así como también de los riesgos a los que se exponen durante la jornada de trabajo, en la mayor parte de estos se denota el interés por dar a conocer los factores asociados a la probabilidad de sufrir un daño corporal o de padecer cualquier tipo de enfermedades laboral.

Tabla 3. Metodología utilizada en artículos analizados.

\begin{tabular}{llll}
\hline \multicolumn{1}{c}{ Metodología } & \multicolumn{1}{c}{ Autores } & $\mathbf{N}^{\circ}$ \\
\hline Revisión & de & & \\
Literatura & Mantva, 2001); (Solís Carcaño, 2006); (Fontaneda, Camino, & \\
& Monte, Riesgos psicosociales en el trabajo y salud ocupacional, & \\
& 2012); (Ministerio de Trabajo y Empleo, 2008); (Viglioglia, 2008); & \\
& (Arias Gallegos W., 2012); (Sanz, 2013); (Pichardo \& Jiménez, & \\
& 2007); (Ramos \& Abuja, 2014); (Guillén, 2014); (Weir \& Lander, \\
\hline
\end{tabular}


2005); (Collado, 2008); (Echeverri Urquijo \& Yepes Palacio, 2011);

(Andrade \& Gómez, 2008).

Estudios (De Vicente Abad, 2006); (Arias Gallegos, 2011); (Calvo, 2014);

Descriptivos

(Arenas \& Cantú, 2013); (Alcantara, 2014); (Mugica, Roman, \&

Cadiz, 2004); (Bellorín, Sirit, Rincón \& Amortegui, 2007); (Arias

Castro \& Martínez Oropesa, 2017); (Instituto Nacional de

Seguridad e Higiene en el Trabajo, 2002); (Cardoso, Martínez, López, \& Arezes, 2013).

Estudios de Casos (Lobato, 2016)

o análisis de

situación

Cualitativo

(Peña, 2018); (Santurio, Ferrera, \& López, 2003).

cuantitativo,

Exploratorio,

descriptivo

correlacional.

De campo y (Arias \& Reyes, 2016).

1

laboratorio

\section{Fuente: Datos obtenidos en la revisión sistemática exploratoria.}

Las Publicaciones de revisión se enfocaron al estudio de los riesgos físicos, enfermedades profesionales y vibraciones. Arias (2012), pone énfasis en los precursores, e indica que los primeros indicios que se registran sobre la salud ocupacional y riesgos laborales datan desde la antigüedad, destacándose los aportes de Hipócrates, Plinio y de Paracelso o Ramazzini durante el periodo del Renacimiento; en tanto que la seguridad industrial ingresa formalmente al marco de la historia a partir de la Primera Revolución Industrial y se institucionaliza con el transcurso de los años.

Para Fontaneda, Camino y Manzanedo del Campo (2005) en el sector de la construcción las condiciones físicas son peores que en la industria; destacando que la formación y el reconocimiento de los trabajadores son menor; así como los sobreesfuerzos en las bajas y que un $52,1 \%$ de los contratos son por obra o servicio. Echeverri y Urquino (2011), identificaron los riesgos en las obras de construcción, a través del estudio de las Condiciones de Seguridad, evidenciando que los riesgos prioritarios son en orden de mayor a menor grado de repercusión: el ruido causado principalmente por los equipos de vibración. En otra investigación se muestra una elevada prevalencia de síntomas musculo - esqueléticos en los trabajadores “[...], lo que orienta al desarrollo de estrategias de reducción y prevención de riesgos a fin de minimizar daños corporales y/o enfermedades incapacitantes". (Bellorín, Sirit, Rincón, \& Amortegui, 2007). Los factores relacionados con el trabajo aumentan el riesgo de sufrir una enfermedad junto con otros factores extra laborales. 
De acuerdo a Lobato (2010) "La valoración de una enfermedad como profesional exige poner en correlación conceptos médicos y legales que permitan determinar la existencia de una patología concreta y su relación con los riesgos presentes en el trabajo" (p. 94). A medida que se toma conciencia del problema, es preciso determinar su alcance, adoptar medidas contundentes y evitar sus consecuencias. (Guillén, 2014).

El sector de la Construcción se ubica en el segundo lugar de mayor Índice de Incidencia de enfermedades profesionales, siguiendo al sector industrial (De Vicente Abad, 2006). El campo aplicable de la ingeniería civil en la mayor parte de las actividades el trabajador se ve expuesto a vibraciones generadas por aplanadoras manuales, maquinaria pesada como rodillo, tractores y retroexcavadoras, etc. (Andrade \& Gómez, 2008).

Las vibraciones según Santurio, Ferrera y López (2003) "Son movimientos oscilatorios de una o varias masas bajo los efectos de diversas solicitaciones" (p.10). Esto se trata de una manifestación de la energía que es traducida en oscilaciones de determinadas partículas que constituyen la materia y que son propagadas desde un foco de origen a través de un medio físico cualquiera. Para Arias, Martínez y Reyes (2016) “Las vibraciones de origen mecánico representan un riesgo para la salud de las personas, cuando éstas se exponen al operar herramientas, equipos o máquinas que generan vibraciones" (p. 28). Desde el punto de vista de la seguridad y Salud en el Trabajo, los trabajadores que se exponen a vibraciones críticas en cuerpo entero, pueden afectar enormemente la salud y el bienestar de los trabajadores expuestos a estos riesgos.

Mujica y Cádiz (2004) estudiaron a 36 operadores de equipos pesados, expuestos a la acción combinada del ruido y las vibraciones con características similares y no expuestas. En los resultados se encontró niveles de ruido y vibraciones que excedían los límites higiénicos tipificados. Se evidenció que en la exposición combinada al ruido y las vibraciones hay alteraciones de la salud que repercuten sobre la esfera psíquica de los trabajadores. Wriu (2012), en su estudio valora los riesgos a los que se exponen los trabajadores y determina los efectos a la salud por la exposición a las vibraciones de las maquinarias y equipos. Existen trabajadores expuestos principalmente a herramientas manuales de vibración, lo que hace que sean candidatos a padecer una serie de enfermedades de la extremidad o dedo blanco o en el desorden vaso espástico, alteraciones de discos en la columna y hernias en conductores de maquinaria pesada, entre otras.

A criterio de Viglioglia (2008), El fenómeno de Raynaud (dedo blanco), se caracteriza por la cesación transitoria del flujo sanguíneo hacia los dedos de manos y pies, que clásicamente se manifiesta por alteración trifásica del color cutáneo. Se instala cuando el fenómeno de 
Raynaud se expresa debido a alguna afección subyacente. Para Calvo (2014) las vibraciones transmitidas a las manos, a diferencia de las vibraciones de cuerpo completo, son aquellas que entran en el cuerpo a través del sistema mano - brazo y se las puede encontrar en diferentes procesos industriales, tales como en la construcción. De acuerdo a Griffin (como se citó en Calvo, 2014) en el comportamiento mecánico de las extremidades superiores frente a la transmisión de vibraciones depende tanto de las magnitudes físicas de la vibración (amplitud, frecuencia y dirección) como de la respuesta biomecánica del sistema mano - brazo.

Cardoso, Martínez, López y Arezes (2013), demuestran que uno de cada cuatro trabajadores está expuesto a vibraciones (ya sean en extremidades superiores o en todo el cuerpo) durante al menos una cuarta parte de la duración de su jornada laboral, provocando trastornos musculoesqueléticos por los efectos de la vibración a la que están expuestas los trabajadores del sector de la construcción, suponen un alto coste social y económico. Ramos y Abuja (2014) investigaron sobre la relación entre Trastornos Músculo - Esqueléticos (TME) y exposición a Vibraciones de Cuerpo Entero (VCE), las zonas donde estos se presentan de acuerdo a los valores registrados y evaluados, y mediante los estadísticos aplicados. Se concluye que si se puede relacionar los TME en zonas lumbares y cervicales con la exposición a VCE.

De acuerdo a Arenas y Cantú (2013) la población estudiada tuvo síntomas sin lesión, las partes más afectadas fueron mano-muñeca derecha con el $65.5 \%$, espalda el $62.2 \%$ y mano muñeca izquierda el $44.2 \%$. La periodicidad de los reconocimientos periódicos, en trabajadores expuestos a vibraciones mano-brazo, deberá establecerse en función del nivel de la aceleración, [...]. En el caso de vibraciones transmitidas al cuerpo entero los reconocimientos médicos periódicos deberían tener una periodicidad anual cuando el límite de exposición diaria.

En otro estudio realizado por El instituto Nacional de Seguridad e Higiene en el Trabajo (2002), determinan que:

Para la vibración transmitida al sistema mano-brazo, el resultado es la suma energética de la vibración ponderada obtenida en cada uno de los canales, en los casos en que no se ha medido en los tres ejes, se supone que los ejes no medidos tienen una vibración del $30 \%$ del eje dominante (punto 6.1.6 de la parte 2 de la norma). (p.6).

El estudio realizado a una población de trabajadores expuestos a las diversas vibraciones, debido a que operan equipos de mayor vibración como son: volquete, perforadora, excavadora y motoniveladoras, se determinó que la dirección en que inciden en el cuerpo humano las vibraciones de maquinarias en promedio no afecta la salud de los trabajadores, porque no 
llegan al mínimo establecido por ley que es el 0,5 m/s2. El tiempo de exposición de $8 \mathrm{~h}$ de las vibraciones de maquinarias en cuerpo entero es de $0,5 \mathrm{~m} / \mathrm{s} 2$ (Alcantara, 2014).

Peña (2018) indica que:

...los trabajadores que se encuentran expuestos a los factores de riesgo como ruido y vibraciones, durante la operación y mantenimiento de grupos electrógenos, en una jornada de 20 días trabajados por mes con 12 horas laborables al día, se les detectó enfermedades de tipos músculo-esqueléticos definidas como alteraciones de columna vertebral y alteraciones en articulaciones (pp. 19-45).

Para lograr una mayor seguridad en la construcción, es importante evaluar los riesgos contenidos en cada tarea y esforzarse en controlar la exagerada exposición, mediante cambios tecnológicos, control del ambiente de trabajo y suministro de equipos de protección individual. Se espera que el aporte de estos estudios contribuya de forma significativa a un mejor conocimiento de las causas que provocan los accidentes laborales en el sector de la construcción y que lleven a una reducción de los mismos.

\section{Discusión}

El presente estudio centra su interés en los cambios que han ocurrido en los últimos años sobre los riesgos físicos y las enfermedades profesionales. Se presentan diversos conceptos sobre factores de riesgo en el trabajo y sus consecuencias, concluyendo con recomendaciones que ayuden a fomentar la salud de los trabajadores como estrategia para mejorar la salud de las personas (Gil Monte, 2012).

A criterio de Collado (2008) "Se considera factor de riesgo de un determinado tipo de daño aquella condición de trabajo, que, cuando está presente, incrementa la probabilidad de la aparición del daño" (p. 4). Desde la mirada del daño ya producido, los factores de riesgo nacen como causas para que se inicien investigaciones sobre los accidentes, mismos que son pertinentes al trabajo realizado ya sea en alturas, labores de excavación, de carácter temporal durante sus jornadas de trabajo, entre otros. (González, Bonilla, Quintero, Reyes, \& Chavarro, 2016).

Velasco (2013) en su estudio sobre Seguridad y Salud en el Trabajo en América Latina y el Caribe, calculan que entre el $50 \%$ y el $70 \%$ de la fuerza laboral en los Países subdesarrollados, se exponen a un sin número de riesgos y peligros que afectan su salud, principalmente los trabajadores de la construcción son los de mayor prevalencia, ya que se exponen a otros peligros físicos como la vibración. Según Sanz (2013): 
Un riesgo que parece ir muy asociado a los trabajadores mayores en la construcción es la pérdida de audición, ya que, en tareas con altos niveles de ruido, como es habitual en la construcción, las consecuencias de la presbiacusia se pueden agudizar en este colectivo" (p. 55).

La exposición a equipos de vibración en construcciones civiles, es una de las causas principales que provocan riesgo físico y enfermedades profesionales. Para Solís (2009) estos se exponen a un sin número de riesgos que pueden tener carácter químico, físico, biológico y social, destacando que es en este sector donde la cultura de la seguridad es muy baja.

Según Weir \& Lander (2005) las herramientas vibratorias portátiles, tales como: taladros, pulidoras, taladros eléctricos, sierras y martillos neumáticos pueden producir Hand arm vibration syndrome. (HAVS), lo que consiste en daños de tipo vascular, neurológicos y músculo-esqueléticos en trabajadores que utilizan este tipo de equipo.

Para Pichardo y Jiménez (2007) las vibraciones en el campo de la higiene, se corresponden a esta detección de frecuencias y aceleraciones y su correlación al tiempo y tipo de exposición que sufre un trabajador, esto implica necesariamente hablar de tiempo de exposición, frecuencia de la vibración en $\mathrm{Hz}$ y aceleración en $\mathrm{m} / \mathrm{s}^{2}$. En ningún momento las vibraciones de forma independiente al material procesado superaron los límites permisibles de exposición. (Arias Castro \& Martínez Oropesa, 2017). Existen un finito número de dificultades al momento de realizar una evaluación de los riesgos laborales tales como: "deficiencias en la transmisión de la información al evaluador, carencias formativas en el evaluador, dificultades de acceso a la información relevante para el sector de actividad, conductas defensivas frente a las responsabilidades en los intervinientes en el proceso, etc.” (Silva Kusi, 2001, p. 83).

La industria de la construcción debe ser muy vigilada, debido a los trabajadores se ven expuesto a un mayor número de riesgos y enfermedades que afectan directa o indirectamente al sistema de salud y al desarrollo económico del país. De acuerdo a Arias (2011) las causas más frecuentes que motivan el desuso de las enfermedades profesionales (EPPs) en los trabajadores de construcción son la falta de comodidad y la dificultad que les genera en la realización de sus labores.

\section{Conclusiones}

En lo que respecta a los riesgos físicos y enfermedades profesionales, estos pueden causar daño a la seguridad y salud de los trabajadores que operan con equipos de vibración en las construcciones civiles. Además, deben contar con la existencia de una planificación para la prevención de los riesgos que se derivan del trabajo, con el fin de disminuir las consecuencias en el caso de que se presente algún tipo de accidente o enfermedad profesional. 
Lo que las máquinas de vibración producen en el cuerpo del trabajador, depende de su aceleración que se transmite a sus manos, causando efectos perniciosos que pueden verse agravados por un ambiente de trabajo frío y húmedo. Las herramientas de golpe neumáticas, de mano, la maquinaria de movimiento de tierras y otras máquinas móviles inducen a los obreros a vibraciones en todo el cuerpo o en una parte del mismo. Además, en ciertas partes del cuerpo se producen lesiones mano-brazo por manipulación de herramientas manuales como compactador, vibrador, taladro, cortadora de césped, concretera, cortadora de maleza, roto martillo, pulidoras entre otras.

\section{Recomendaciones}

Se recomienda capacitación periódica, seguimiento, y conductas enfocadas al autocuidado, inspecciones de seguridad, y mejoramiento de las buenas condiciones higiénicas.

Determinando las afecciones provocadas por las vibraciones, se debe usar equipos de protección personal para salvaguardar la integridad de los colaboradores de las empresas de construcción y con esto, reducir los riesgos en los sectores más vulnerables como lo son los obreros.

\section{Referencias Bibliográficas}

Alcantara, M. (2014). 6.27. Estudio de la vibración producida por maquinaria minera en la salud de los trabajadores en la Unidad Minera Breapampa. Obtenido de http://repositorio.uni.pe/bitstream/uni/1216/1/valdivieso_gl.pdf

Alcantara, M. (2014). Estudio de la vibración producida por maquinaria minera en la salud de los trabajadores en la Unidad Minera Breapampa. Obtenido de http://repositorio.uni.pe/bitstream/uni/1216/1/valdivieso_gl.pdf

Andrade, V. y Gómez, I. (25 de abril de 2008). Salud Laboral. Pensamiento Psicológico, $4(10), 9-25$.

Arenas, L. y Cantú, O. (mayo de 2013). Factores de riesgo de trastornos músculo-esqueléticos crónicos laborales. Medicina Interna México, 29(4), 370-378.

Arias Gallegos, W. (2011). Uso y Desuso de los Equipos de Protección Personal en Trabajadores de Construcción. Ciencia \& Trabajo, 119-124.

Arias Gallegos, W. (2012). Revisión histórica de la salud ocupacional y la seguridad industrial. Revista Cubana de Salud y Trabajo. 13(3), 45-52.

Arias, G. M. y Reyes, C. (2016). Evaluación de las vibraciones globales transmitidas a trabajadores en una empresa agroindustrial productora de azúcar. Salud Trabajadores. 24(1), 27-37, 27-37. 
Bellorín, M., Sirit, Y., Rincón, C., \& Amortegui, M. (2 de julio-diciembre de 2007). Síntomas Músculo Esqueléticos en Trabajadores de una Empresa de Construcción Civil. Obtenido de https://www.redalyc.org/pdf/3758/375839287003.pdf

Calvo, D. (2014). Enfermedades osteoarticulares o angioneuróticas provocadas por la vibraciones mecánicas. Ciencia forense, 53-64.

Cardoso, J., Martínez, M., López, M. y Arezes, P. (2013). Efectos sobre la salud de los trbajadores derivados de la vibracion producida por el manejo de maquinaria en el sector de la construcción. El Ageo, 1-8.

Collado, L. (2008). Prevención de riesgos laborales: Principios y Marco Normativo. Obtenido de http://www.ehu.eus/ojs/index.php/rdae/article/viewFile/11447/10573

Constitución. (2008). en su Título VI "Régimen de Desarrollo", Capítulo Sexto, Sección Tercera -Formas de trabajo y su retribución, Art. 326, Numeral 5. Montecristi, República del Ecuador: Asamblea Nacional.

De Vicente Abad, M. (2006). Enfermedades Profesionales en el sector de la Construcción, periodo 2002-2006. España: Instituto Nacional de Seguridad e Higiene en el Trabajo .

Echeverri Urquijo, H., \& Yepes Palacio, D. (enero-junio de 2011). Factores de riesgo en obras de construcción del Área Metropolitana del Valle de Aburrá- Colombia. Revista Politécnica ISSN 1900-2351. Año 7 número 12, 71-80. Obtenido de http://revistas.elpoli.edu.co/index.php/pol/article/view/181/155

Fontaneda González, I., Camino López, M., Manzanedo del Campo, M. y Sáiz, R. M. (9 de septiembre de 2005). La prevención de riesgos en el sector de la construccion en España. IX Congreso de Ingeniería de Organización. Gijón: Dpto. de Ingeniería Civil. Escuela Politécnica Superior Universidad de Burgos. Obtenido de http://adingor.es/congresos/web/uploads/cio/cio2005/prevencion_riesgos//118.pdf

Gil Monte, R. (2012). Riesgos psicosociales en el trabajo y salud ocupacional. Rev Peru Med Exp Salud Publica. 29(2):237-41, 237-241.

Gómez Gerreora, R. (2015). Condiciones de trabajo y salud en el sector de la contrucción. ?Cuestiones de Jerarquíias?. España: UPNA.

González, A., Bonilla, J., Quintero, M., Reyes, C. y Chavarro. (2016). Análisis de las causas y consecuencias de los accidentes laborales ocurridos en dos proyectos de construcción. Rev. ing. constr. 31(1), 5-16.

Guillén, C. (2014). El desafío de la gestión de las enfermedades profesionales. Solvitas perambulum. Medicina y Seguridad del Trabajo (1), 144-156.

INSHT. (1995). Ley 31/1995, de Prevención de Riesgos Laborales. Prevención de Riesgos Laborales (26), 10-11.

Instituto Nacional de Seguridad e Higiene en el Trabajo. (2002). Arriesgarse para no perder el empleo: las secuelas en la salud de los obreros de la construcción del Mercosur. Nipo 792-11-087-6. Mediciones realizadas por AAC Centro de Acústica Aplicada, SL., 1309. 
Kitchenham, B. (2004). Procedures for performing systematic reviews. Keele, UK, Keele University, 33(2004), 1-26.

Lobato, J. (2016). Calificación y valoración de la enfermedad profesional: Análisis de la situación. Obtenido de http://scielo.isciii.es/pdf/mesetra/v62sextra/ponencias8.pdf

Luttmann, A., Jarger, M. y Griefahn, B. (2004). Prevencion de trastornos musculoesqueléticos en el lugar de trabajo. Prevencion de trastornos musculoesqueléticos en el lugar de trabajo $(O M S)(5), 1-31$.

Machado, R., Tamames, S., López, M., Mohedano, L., D’Agostino, M., \& Veiga, J. (2009). Revisiones sistemáticas exploratorias. Med. segur. trab. vol.55 no.216 Madrid, 12-19.

Manchado Garabito, R., Tamames Gómez, S., López González, M., Mohedano Macías, L., \& Veiga de Cabo, J. (2009). Revisiones sistemáticas exploratorias. Medicina y seguridad del trabajo, 55(216), 12-19.

Manchado, R., Tamames, S., López, M., Mohedano, L., D’Agostino, M. y Veiga, J. (2009). Revisiones sistemáticas exploratorias. Med. segur. trab. vol.55 no.216 Madrid, 12-19.

Merino Trujillo, A. (2011). Como escribir documentos científicos (Parte 3). Artículo de revisión. Salud en Tabasco, 17(1-2), 36-40.

Ministerio de Trabajo y Empleo. (10 de Enero de 2008). Registro Oficial N²49. Reglamento de Seguridad y Salud para la Construcción y obras Públicas. Quito, Pichincha, Ecuador. Recuperado el 15 de diciembre de 2018, de http://www.trabajo.gob.ec/wpcontent/uploads/downloads/2012/12/Reglamento-de-Seguridad-y-Salud-para-laConstrucci \%C3 \%B3n-y-Obras-P \%C3 \%BAblicas.pdf

Mugica, J., Roman, H. y Cadiz, A. (2004). Efectos del ruido y las vibraciones en operaciones de equipos pesados. Revista Cubana de Salud y Trabajo, 5(1), 39-47. Obtenido de http://www.bvs.sld.cu/revistas/rst/vol5_01_04/rst07104.pdf

Peña, M. (2018). Ruido y vibraciones ene el ambiente laboral y su incidencia en la salud de los trabajadoresa durante la operacion y mantenimiento de grupos electrógenos. Ambato, República del Ecuador: Universidad Técnica de Ambato. Obtenido de http://repositorio.uta.edu.ec/bitstream/123456789/28550/1/Tesis_\%20t1460mshi.pdf

Pichardo, G. y Jiménez, M. (2007). Vibraciones y salud en el trabajo. Exposiciones virtuales, $1-16$.

Ramones, G. (diciembre de 2010). Evaluación de la carga postural y síntomas musculo esqueléticos en trabajadores de las construcción. Venezuela: Universidad de Zuliia. Obtenido de http://tesis.luz.edu.ve/tde_arquivos/104/TDE-2011-11-01T09:39:15Z2074/Publico/ramones_grisell.pdf

Ramos, C. y Abuja, G. (2014). Exposición a vibraciones de cuerpo entero y trastornos musculoesqueléticos en operaciones de maquinarias pesada en obra civil. 1-5.

Santurio, J., Ferrera, A. y López, V. (2003). Exposición a vibraciones globales en maquinaria de obra pública. Estudio de situación. España: Universidad de Oviedo. 
Sanz, F. (2013). Estudio sobre riesgos laborales emergentes en el sector de la construcción. Instituto Nacional de Seguridad e Higiene en el Trabajo (INSHT), 1-145.

Silva Kusi, M. (10 de julio-diciembre de 2001). Los riesgos del trabajo en la construcción. Los casos de Rosario y Montevideo. Obtenido de 292-319.

Social, M. d. (2014). Enfermedad Profesional. Instituto de Seguridad Laboral, 1-3. Obtenido de https://www.isl.gob.cl/wp-content/uploads/2014/04/Enfermedad_Profesional.pdf

Solís Carcaño, R. (29 de junio de 2006). Riesgos en la salud de los trabajadores de la construcción. Ingenieria, 10(2), 67-74.

Valenzuela, W. (2012). Efectos a la salud por exposicion a vibraciones. Prevencionar.com, 722.

Velásco Mayor, I. (2013). Incidencia de accidentes laborales y enfermedades ocupacionales en la constructora Miguel Angel Auad y diseño de un sistema de gestión de riesgo de trabajo para la implementación del Sart. Guayaquil, República del Ecuador: Universidad de Guayaquil. Obtenido de http://repositorio.ug.edu.ec/bitstream/redug/7939/3/TESIS.pdf

Viglioglia, P. (2008). Fenómeno y síndrome de Raynaul. Act Terap Dermato, 18-21.

Weir, E. y Lander, L. (2005). Hand arm vibration syndrome. . Canadian Medical Association Journal, 172(8), 1001-1002. . 